\title{
Torulaspora delbrueckii
}

National Cancer Institute

\section{Source}

National Cancer Institute. Torulaspora delbrueckii. NCI Thesaurus. Code C125962.

A species of ascomycetous yeast in the Torulaspora genus that has the ability to ferment and assimilate carbon compounds. 